\title{
Effect of Bias Point and Modulation Index of Mach-Zehnder Modulator on the Performance of Coherent Optical OFDM in Nonlinear and Dispersive Fiber Channels
}

\author{
Mohammed Salah-El-Din Abdullah, Ahmed K. Sultan Salem, and Hossam M. H. Shalaby \\ Electrical Engineering Department, Faculty of Engineering, Alexandria University \\ Alexandria 21455, Egypt \\ mohammed_salah_1985@yahoo.com, salatino@stanfordalumni.org, shalaby@ieee.org
}

\begin{abstract}
Coherent detection can be used for optical OFDM where the received signal is mixed with a locally generated carrier. We study the effect of the DC bias point and the modulation index of Mach-Zehnder modulator on the BER performance of coherent optical OFDM.
\end{abstract}

\section{Introduction}

This paper focuses on the coherent flavor of optical OFDM. Coherent optical OFDM with direct up/down conversion is briefly discussed and the system architecture is described in Section II. COOFDM was proposed to combat chromatic dispersion and fiber polarization mode dispersion (PMD). In Section II, we focus on one component of the system: the Mach-Zehnder modulator (MZM) and its nonlinearity. The nonlinearity analysis for RF-to-optical up-converter has been performed in [1]. However, the effect of nonlinearity on the bit error rate (BER) has not been addressed. We discuss the effect of the DC bias point of the MZM and the modulation index $(M)$ of the MZM and the nonlinearity they introduce on the BER performance of the system. A mathematical analysis for the system is given in Section III. In Section IV, a simulation of the system is presented. In Fig. 2, the BER is plotted vs. $M$ for fixed DC bias to show the optimal value of $M$. We conclude the paper in Section $\mathrm{V}$, by finding and the optimal value of the modulation index.

\section{Mach-Zehnder Modulator}

In this section, we study the transfer characteristics of MZM. The optical signal at the output of the RFto-optical up-converter is

$$
\begin{gathered}
E(t)=\exp \left(\omega_{L D 1} t+j \varphi_{L D 1}\right) \cdot E^{B}(t) \\
E^{B}(t)=\cos [(\mathrm{M} / 2) \operatorname{Re}[x(t)]+\varphi / 2]+\mathrm{j} \cos [(M / 2) \operatorname{Im}[x(t)]+\varphi / 2]
\end{gathered}
$$

Signal $x(t)$ is the complex output of the RF OFDM transmitter and is normalized so that $E\left\{|x(t)|^{2}\right\}=$ 1. Parameter $M$ is the modulation index of the $\mathrm{MZM}, \varphi=V_{\mathrm{DC}} \pi / V_{\pi}$ is a static phase shift that depends on the DC bias, and $E^{B}(t)$ is the baseband version of the optical field $E(t)$.

Voltage $V_{\mathrm{DC}}$ is the DC bias of MZM, whereas $V_{\pi}$ is the half-wave switching voltage. Parameters $\omega_{L D 1}$ and $\varphi_{L D 1}$ are the frequency and phase of laser transmitter, respectively.

The analysis given in [1] is restricted to the case of two complex subcarrier tones. Here, the analysis is more general, allowing all OFDM subcarriers to carry data. Also, the effect of the bias point on BER as a performance metric has not been examined despite its primacy in the analysis and assessment of the performance of digital communication systems. We verified, through simulations, that the bias point that gives the best BER performance is the null-bias point as suggested in [1]. We also present the optimal value for the modulation index $M$ based on the simulations.

\section{Mathematical Analysis}

The optical field at the input of the optical fiber channel is given by (1) and (2).

The quadrature homodyne receiver [2] shown in Fig. 1 is used.

Following the analysis presented in [2], the received photocurrents at the upper and lower balanced receivers, excluding shot noise and ignoring the channel effect, for simplicity, are respectively:

$$
\begin{gathered}
i_{I}(t)=(R / 4)\left|E_{o}(t)+A_{L} e^{j \omega_{L O} t}\right|^{2}-(R / 4)\left|E_{o}(t)-A_{L} e^{j \omega_{L O} t}\right|^{2}=R A_{L} \operatorname{Re}\left\{E_{B}(t)\right\} \\
=R A_{L} \cos [(M / 2) \operatorname{Re}[x(t)]+\varphi / 2] \\
i_{Q}(t)=(R / 4)\left|E_{o}(t)+A_{L} e^{j \omega_{L O} t+j \pi / 2}\right|^{2}-(R / 4)\left|E_{o}(t)-A_{L} e^{j \omega_{L O} t+j \pi / 2}\right|^{2}=R A_{L} \operatorname{Im}\left\{E_{B}(t)\right\} \\
=R A_{L} \cos [(M / 2) \operatorname{Re}[x(t)]+\varphi / 2]
\end{gathered}
$$




\section{JWA4.pdf}

where $R$ is the photodetector's responsivity. Here it assumed that the local oscillator's frequency is equal to the optical carrier's frequency.

In the simulation, the optical fiber channel was modeled by solving the nonlinear Schrödinger using the split-step Fourier method taking into account dispersion and fiber nonlinearities. If the MZM is biased at the null-point, and $M$ is small enough (below $0 \mathrm{~dB}$ ), then the nonlinearity introduced by the transfer characteristics of the MZM can be ignored.

After RF demodulation, the received OFDM samples are:

$$
y=y_{I}+j y_{Q}=\frac{R A_{L} M}{4} z
$$

where $z$ is the baseband OFDM signal, and $\omega_{R F}$ is the RF carrier frequency.

A simplified formula for the electrical signal-tonoise ratio per subcarrier can be obtained:

$$
\mathrm{SNR}_{\text {sub. }}=E\left[\frac{|y|^{2}}{2 \sigma^{2}}\right]=\frac{R^{2} A_{L}^{2} M^{2}}{32 \sigma^{2}}
$$

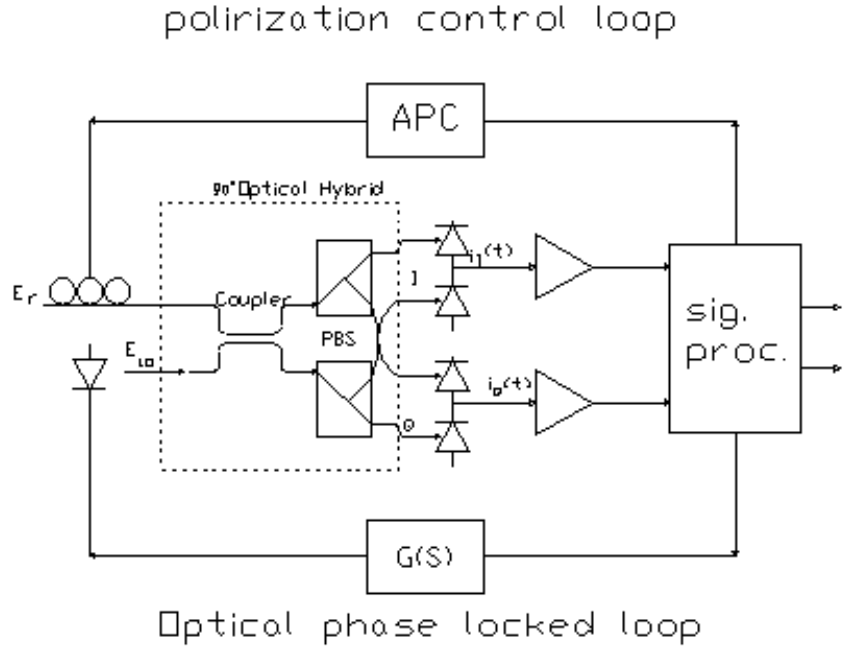

Fig. 1. Quadrature homodyne receiver

For QPSK modulation the BER is:

$$
P_{e} \cong \operatorname{erfc}\left(\sqrt{\mathrm{SNR}_{\text {sub } . / 2}}\right)=\operatorname{erfc}\left(\sqrt{R^{2} A_{L}^{2} M^{2} / 64 \sigma^{2}}\right)
$$

\section{Simulation Model}

The CO-OFDM system is simulated assuming QPSK modulation. The OFDM symbol period is $25.6 \mathrm{~ns}$ and 128 subcarriers are used. The guard interval is equal to one quarter of the observation period. Parameter $V_{\pi}$ is set to $1 \mathrm{~V}$ and $V_{\mathrm{DC}}$ is taken equal to $1 \mathrm{~V}$ (corresponding to the null point). The optical channel is modeled by solving the nonlinear Schrödinger equation using the split-step Fourier method. This takes into account the dispersive and nonlinear effects of the fiber. The nonlinear index coefficient $n_{2}=6 \times 10^{-13}[1 / \mathrm{mW}]$, the fiber loss $\alpha_{\mathrm{dB}}=$ $0.2 \mathrm{~dB} / \mathrm{km}$, and the dispersion parameter $D=2 \mathrm{ps} /(\mathrm{km}-\mathrm{nm})$. The propagation distance is $1000 \mathrm{~km}$. In order to compensate for the attenuation, a preamplifer (optical amplifier) with a gain of $200 \mathrm{~dB}$, is used at the receiver. The thermal noise of the receiver is modeled as AWGN. Shot noise is also considered in our model and is modeled as a Poisson process. The receiver strips the cyclic prefix, performs the FFT operation, then ideal channel equalization is performed. Finally, the BER is plotted versus the modulation index (M) for null-point bias.

It can be noticed from Fig. 2, that, up to a certain limit, as $M$ is increased, the BER decreases. This is due to the fact that increasing $M$ is equivalent to an increase in the SNR for a fixed noise power as evident from (6), and thus gives better BER performance. Then, $M$ reaches a certain optimal value above which the BER increases as $M$ is increased. As $M$ is increased beyond the optimal point, the signal is affected more by the nonlinear characteristics of the MZM and thus the BER rate performance deteriorates due to the nonlinearities introduced by the MZM. Fig. 2 shows that the optimal value of $M$ is approximately -4.5 $\mathrm{dB}$.

\section{References}

[1] W. Shieh, H. Bao, and Y. Tang, "Coherent optical OFDM: theory and design," Opt. Express 16, 841-859 (2008)

[2] K.-P. Ho, Phase-Modulated Optical Communication Systems (Springer, New York, 2005), pp.66-68.

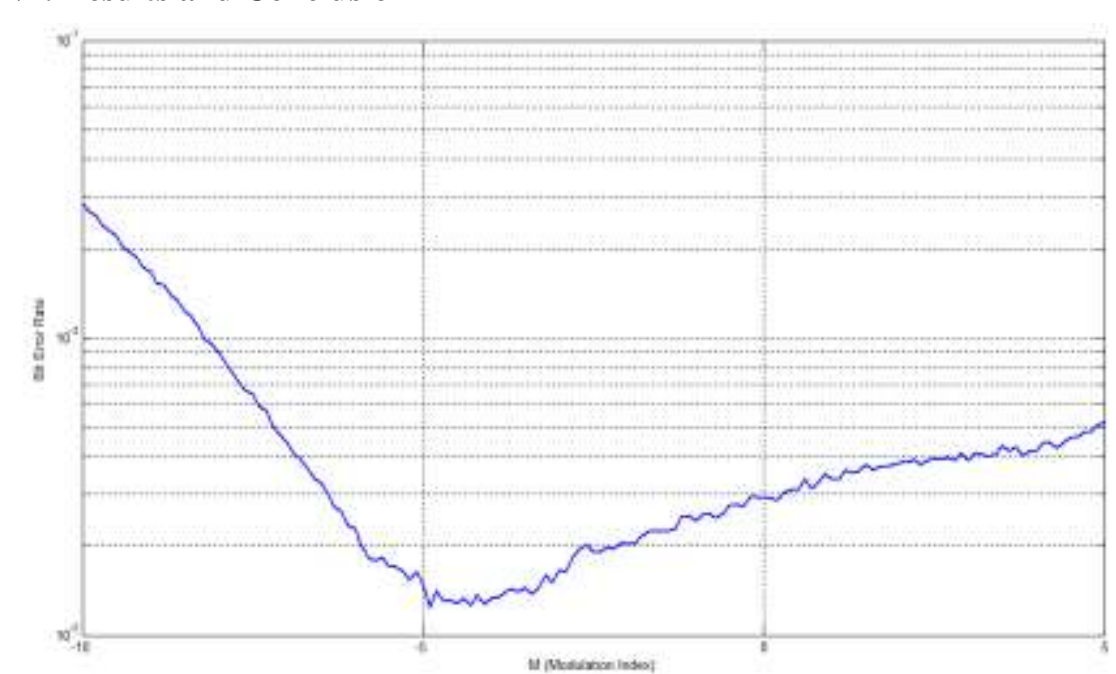

Fig. 2. BER versus modulation index $(\mathrm{M})$ at $\mathrm{V} \_\mathrm{dc}=\mathrm{V} \_\pi$ (null-point), showing an optimal value for $\mathrm{M}$. 